\title{
Nasal RAM cannulae for breathing support and inhalation anaesthesia in infants during videolaryngoscopy.
}

\author{
Andrzej Piotrowski ${ }^{1}$, Teresa Oleniacz ${ }^{2}$ Tomasz Grochowski², Szymon Nalepa ${ }^{1}$. \\ Departments of Anaesthesia and Intensive Care ${ }^{1}$ and Otolaryngology ${ }^{2}$, Children's \\ Memorial Health Institute, Warsaw, Poland.
}

Background and Goal of Study: The safe anaesthesia for direct laryngoscopy must provide sufficient oxygen and ventilation support with no collision with otolaryngologist. On the other hand induction of anaesthesia with $100 \%$ oxygen results in the development of atelectasis within minutes of anaesthetic administration. The use of continuous positive airway pressure (CPAP) during induction may be advantageous - provides a longer duration of apnoea before arterial desaturation occurs, especially in patients with respiratory problems [1]. CPAP is widely used in neonates with obstructive apnea, respiratory distress syndrome, chronic lung disease. The RAM device, invented for nasal ventilation support [2] can be attached to an anesthesia machine and provide both CPAP, and delivery of sevoflurane.

Materials and Methods: Direct videolaryngoscopy was undertaken in 5 infants and 1 child with stridor or unsuccessful extubation. Patients were anesthetized with a combination of sevoflurane in air/oxygen and boluses of i.v. propofol. For maintenance of anesthesia and ventilation assistance sevoflurane (1,0-1,5 MAC) was delivered through the RAM cannulae attached to the Mapelson $D$ circuit. Standard monitoring including ECG, SpO2 and NIBP were used. Nasal CPAP technique was tried, manual breaths given when required. Results and Discussion: Patients with weight from 3,0 to $8,0 \mathrm{~kg}$ were successfully anesthetized with inhalation of sevoflurane through RAM cannulae, only 2 required i.v. doses of propofol. Paracetamol and fentanyl $(0,5 \mathrm{mcg} / \mathrm{kg})$ were used for analgesia. In one case i.v. lidocaine was also administered. There was an efficient anaesthesia level and proper gas exchange during procedures. Two out of 3 intubated patients could be extubated and remained on nasal CPAP afterwards. This kind of delivery of anaesthetic mixture was very practical in an opinion of otolaryngologists.

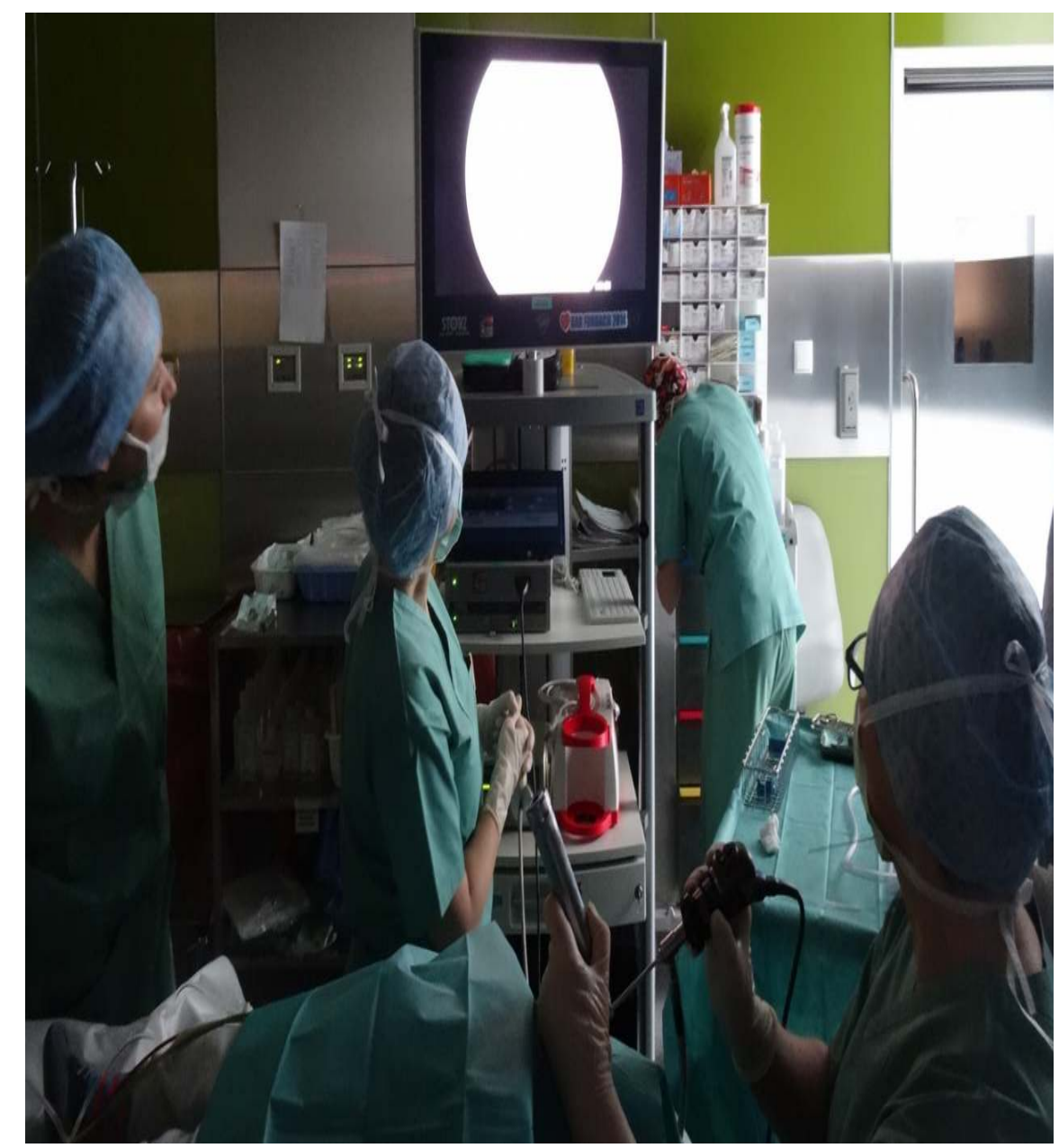

Conclusion:The use of RAM nasal cannulae is useful for gas delivery and sevoflurane anesthesia delivery during videolaryngoscopy in infants.
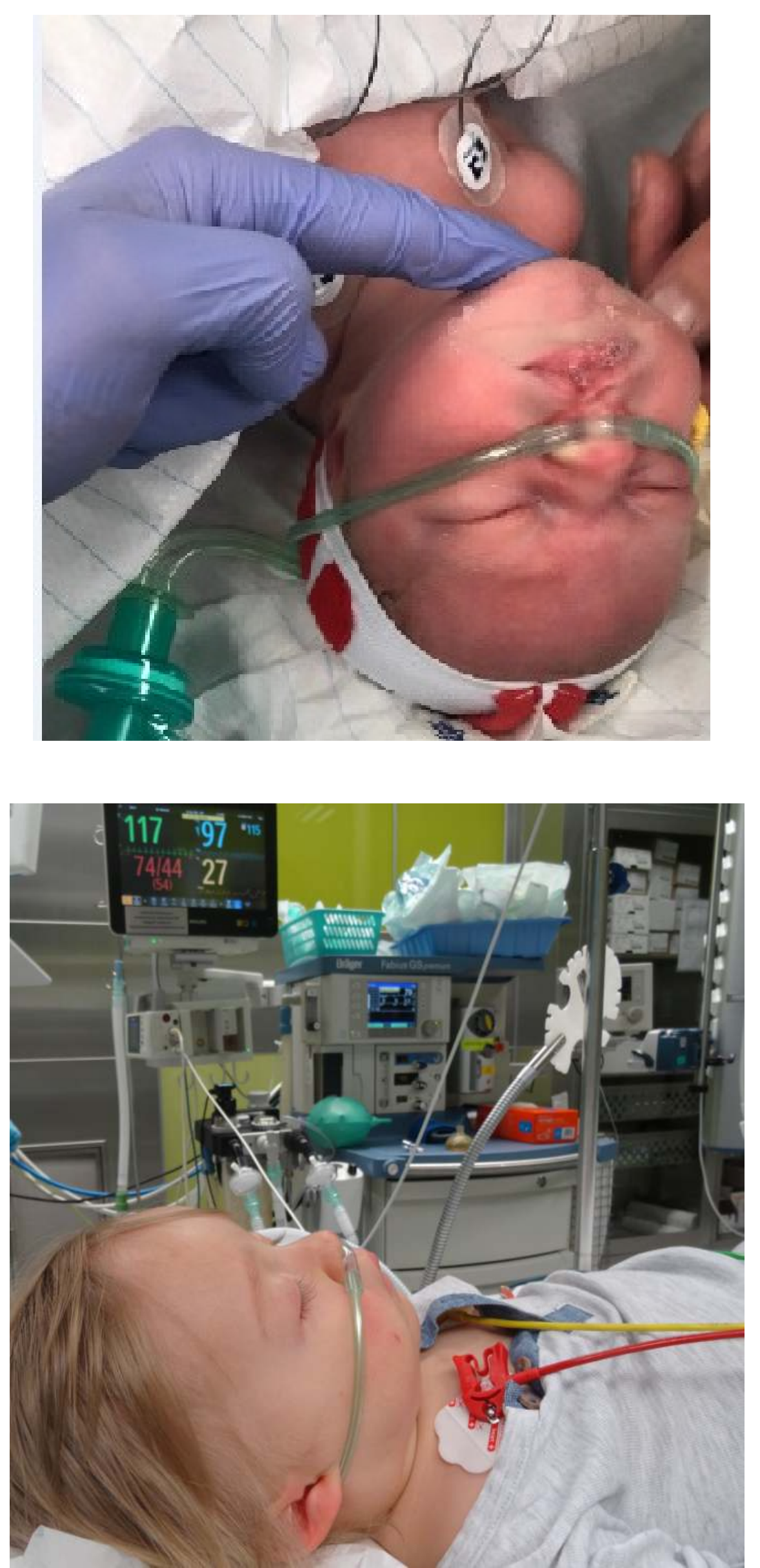

\section{Literature:}

1. Herriger A, Frascarolo P, Spahn DR, Magnusson L. The effect of positive airway pressure during pre-oxygenation and induction of anaesthesia upon duration of non-hypoxic apnoea. Anaesthesia. 2004 Mar;59(3):243-7.

2. Nzegwu NI, Mack T, DellaVentura R. et al. Systematic use of the RAM nasal cannulae in the Yale-New Haven Children's Hospital Neonatal Intensive Care Unit: a quality improvement project. J Maternal Fetal Neonat Med. 2015; 28: 718-21. 